\title{
CELLA MEDIA DISTANCE IN HUMAN BRAIN IN RELATION TO AGE AND GENDER
}

\author{
Lydia Ragan $^{\mathrm{a}}$, Iveta Waczulikova ${ }^{\mathrm{b}}$, Ladislav Guller ${ }^{\mathrm{c}}$, Jozef Bilicky ${ }^{\mathrm{d}}$, Jozef Benuska ${ }^{\mathrm{c}}$
}

${ }^{a}$ Department of Anatomy, Institute of Basic Medical Sciences, Faculty of Medicine, University of Oslo, Norway,

${ }^{b}$ Division of Biomedical Physics, Department of Nuclear Physics and Biophysics, Faculty of Mathematics, Physics \& Informatics, Comenius University in Bratislava, Slovakia,

c Institute of Anatomy, Faculty of Medicine, Comenius University in Bratislava, Slovakia

${ }^{d} 1^{s t}$ Radiology Clinic, Faculty of Medicine, Comenius University in Bratislava

e-mail: lydia.ragan@medisin.uio.no

Received: October 10, 2008; Accepted: November 27, 2009

Key words: Cella media distance/Lateral ventricles/Volume/Brain/Morphometry

Aims: To determine whether simple linear measurements can be used as an accurate and reproducible replacement of the volumetric ones.

Methods: We measured the cella media distance (CM1) and the distance between the right and left human brain surfaces (CM2) - measured along the CM1 line - in the groups of women (F) and men (M) who were divided according to diagnoses into three subgroups (F1-3 and M1-3). Examinations were carried out under standardized conditions: axial serial CT (Computed Tomography) images, in $4 \mathrm{~mm}$ layers (333 patients). Measured values were detected by Osiris Software. Recorded values were statistically analysed.

Results: We found very highly significant (subgroups M1, M3), and significant (subgroups F1, F2, F3, M2) associations between the cella media distance and the decade of age. There were only non-significant differences in CM1 distances between men and women and between the diagnoses groups as well. Correlation between cella media distance and volume of lateral ventricles was greater in men in both, subgroup M1 $(r=0.659, \mathrm{P}<0.0001)$ and in a mixed group where all the three male subgroups M1-3 were combined into one group ( $\mathrm{r}=0.675, \mathrm{P}<0.0001)$. Among women the correlation was lower, however still significant $(r=0.357, \mathrm{P}<0.0001$ for $\mathrm{F} 1$ and $\mathrm{r}=0.465, \mathrm{P}<0.0001$ for $\mathrm{F} 1-3)$.

Conclusions: The cella media distance is much better predictor of brain lateral ventricular volume in men than in women.

\section{INTRODUCTION}

It has already been shown that computed tomography (CT) and magnetic resonance imaging (MRI) can be used to measure brain atrophy that is an important anatomical change in the course of several disease ${ }^{1-4}$, as well as in normal aging ${ }^{5-7}$.

Simple linear measurements taken on axial CT slices were used from the advent of imaging technologies; however, different authors have used different exclusion criteria for sample material and for image analysis as well ${ }^{8}$. Some authors used axial CT images and linear measures to assess cerebral lateral ventricular asymmetry and its possible association with neuropsychiatric disorders 9 . Several investigators have also tried to assess the degree of correlation between some of linear ratios and volume of subcortical structures ${ }^{10}$. However, data regarding correlation between linear and volumetric parameters are still missing.

\section{SUBJECTS, MATERIAL AND METHODS}

\section{Subjects and material}

Quantitative analysis presented in this study has been performed on the image material obtained from a large PACS database of $1^{\text {st }}$ Radiology Clinic, University Hospital in Bratislava. A great amount of CT documentation of all the patients who underwent $\mathrm{CT}$ examination during the period from January $1^{\text {st }} 2005$ by December $31^{\text {st }} 2006$ was reviewed. A data set of 333 subjects (aged 18-96) with normal radiological findings was selected - a set which included both, subjects with and without psychiatric and neurological diagnoses and subjects with head injury in anamnesis as well. Based on consultation with neorologist, the selected subjects were divided according to diagnoses into three groups: Group 1) 223 subjects (133 women or subgroup F1 aged 19-91, and 90 men or subgroup M1 aged 18-92) without any diagnosis that could influence the size of cerebral ventricles or that of the brain; Group 2) 66 subjects (33 women or subgroup F2 aged 20-86, and 33 men or subgroup M2 aged 19-96) without any psychiatric diagnosis or serious neurological diagnosis but with head injury in anamnesis; Group 3) 44 subjects ( 20 women or subgroup F3 aged 21-82, and 
24 men or subgroup M3 aged 26-85) with psychiatric or neurological diagnoses with possible influence on the size of cerebral ventricles or that of the brain. Exclusion criteria for group 1 were as follows: any kind of dementia, any kind of epilepsy, any degenerative nervous system disease, head injury in anamnesis, stroke in anamnesis. To make it simple we can also say that we have analysed a group of women $(\mathrm{F}, \mathrm{n}=186)$ and a group of men $(\mathrm{M}, \mathrm{n}=147)$, divided according to diagnoses into three subgroups each: F1, F2, F3 and M1, M2, M3, respectively.

\section{Methods}

\section{Computed Tomography}

All patients had transverse scanning of the brain performed on a multi-slice CT device (Siemens Somatom Sensation 16). The images covered the entire brain from the vertex to the foramen magnum; final slice thickness was $4 \mathrm{~mm}$ (the number of images per person ranged from 30 to 34 ). Images were obtained parallel to the orbitomeatal line (Fig. 1a). Contrast enhancement was not used in any case. Images were saved on an optical disc, later being transferred to a TravelMate C302XMi Tablet PC Edition and then analysed quantitatively.

\section{Image Analysis}

Transverse CT images were transferred to a TravelMate C302XMi Tablet PC Edition and analysed quantitatively. Quantitative image analysis was performed blind to clinical information, by a skilled observer with a good knowledge of anatomy of the central nervous system. For quantitative image analysis Osiris Software, version 4.19 (University Hospital of Geneva, Switzerland) was used. To measure linear parameters (distances CM1 and CM2, i.e., the $1^{\text {st }}$ and the $2^{\text {nd }}$ distance measured at the level of cella media) Osiris Software functionality called Calliper, that allows distance measurements to be made between two points, was used. CM1 (cella media distance) provides the minimum linear distance between lateral walls of lateral ventricles in cella media region, while the CM2 distance was measured as the width of the brain along the same line as the CM1 distance was (Fig. 1b). To get volume of lateral ventricles both, the Osiris Software functionality called Pencil, and the Tablet Edition computer's electronic pen were used, and this arrangement, giving much more precise results as when using mouse, was really helpful since lateral ventricles had to be outlined manually. The Osiris software calculated the volume of left and right ventricles by multiplying area of ventricle by slice thickness, on each slice ventricles were present. Calculated measures were plotted against age.

\section{Statistical support}

Association between the median of the minimum linear distance between lateral walls of lateral ventricles in cella media region (CM1 distance) and the decade of age was assessed by the Grouped linear regression analysis and Kendall's rank correlation coefficient, separately for each of the subgroups: F1, F2, F3, M1, M2, and M3. Correlation between CM1 distance and volume of lateral ventricles was statistically analysed using simple and multiple linear regression analyses separately for subgroups F1 and M1 as well as for female and male groups as a whole, F1-3 (F1 + F2 + F3) and M1-3 (M1 $+\mathrm{M} 2+\mathrm{M} 3)$, respectively. Pair-wise comparisons across subgroups F1 to F3 as well as M1 to M3 were performed using Tukey approach. Paired Student's t test was used to assess differences between gender subgroups. The threshold value for statistical significance was set at 0.05 .
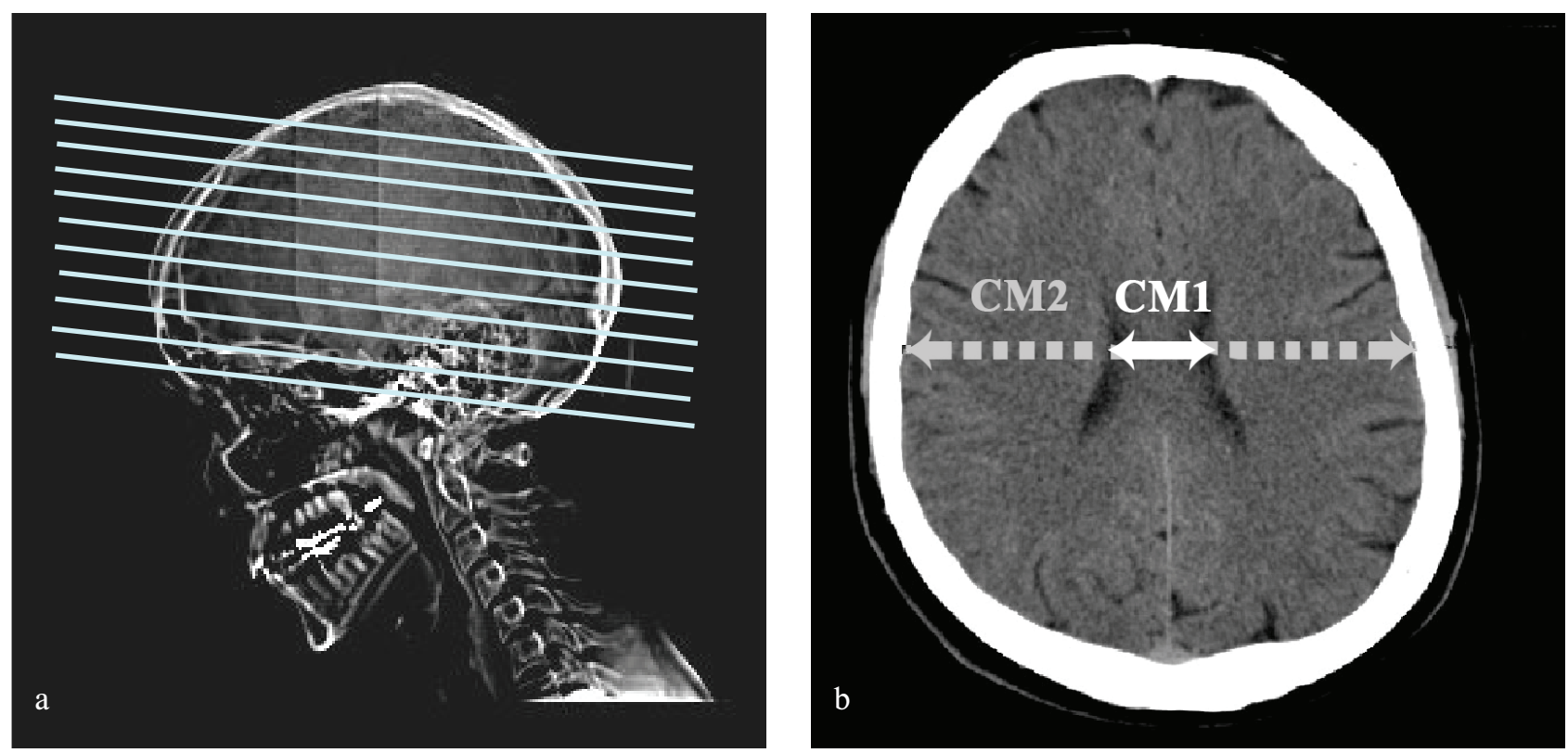

Fig. 1. a) CT images were obtained parallel to the orbitomeatal line, b) CM1 (cella media distance) provides the minimum linear distance between lateral walls of lateral ventricles in cella media region, CM2 distance was measured as the width of the brain along the same line as the CM1 distance was. 


\section{RESULTS}

The results of the CM1 measurement in the female subgroup 1 (F1) which was the most numerous subgroup in this study are summarized in Table 1 . The median of CM1 distance in this subgroup ranged from $21.5 \mathrm{~mm}$ in the $2^{\text {nd }}$ decade to $30.9 \mathrm{~mm}$ in the $8^{\text {th }}$ decade to decrease finally to the value of $25.2 \mathrm{~mm}$ in the $10^{\text {th }}$ decade of age where we had just two measurements. The median of CM1 distance in the male subgroup 1 (M1) ranged from $25.4 \mathrm{~mm}$ in the $3^{\text {rd }}$ decade to $35.3 \mathrm{~mm}$ in the $9^{\text {th }}$ decade of age and the results for this subgroup are summarized in Table 2. In the female subgroup 2 (F2) the median of CM1 distance ranged from $26.75 \mathrm{~mm}$ in the $3^{\text {rd }}$ decade to $29.4 \mathrm{~mm}$ in the $9^{\text {th }}$ decade of age while in the male subgroup 2 (M2) it was from $23.8 \mathrm{~mm}$ in the $3^{\text {rd }}$ decade to $28.7 \mathrm{~mm}$ in the ${ }^{\text {th }}$ decade of age (see Fig. 2b). In group 3 we have noticed an increase in CM1 distance as follows: in the female subgroup 3 (F3) the median ranged from the value of $27.75 \mathrm{~mm}$ in the $4^{\text {th }}$ decade to the value of $34.05 \mathrm{~mm}$ in the $9^{\text {th }}$ decade of age. In the male subgroup 3 (M3) the median of CM1 distance ranged from the value of $24.7 \mathrm{~mm}$ in the $3^{\text {rd }}$ decade to the value of $36.1 \mathrm{~mm}$ in the $9^{\text {th }}$ decade of age (see Fig. 2c). The minimum linear distance between lateral walls of lateral ventricles in cella media region was found to increase with advancing age in each of the three female as well as male subgroups (Fig. 2a, 2b, 2c). There were found very highly significant (subgroups M1, M3), and significant (subgroups F1, F2, F3, M2) associations between the median of the cella media distance and the decade of age (Table 3).

Comparing male and female results it was found out that in each of the three groups differing in diagnoses group 1 (comprising F1 and M1), group 2 (comprising F2 and M2) and group 3 (comprising F3 and M3) - there was slightly higher increase in CM1 distance with age in men compared to that recorded in women (Fig. 2a, 2b, 2c). However, statistical analysis performed separately for groups 1, 2 and 3 has revealed only non-significant differences in CM1 distance between men and women - in each of the three groups (Table 4).

Pair-wise comparisons across the subgroups F1 to F3 as well as M1 to M3 have revealed only non-significant differences in CM1 distance between the predefined subgroups (Table 5, see also Fig. 3a and 3b).

In analyses made separately for both, the subgroup F1 (M1) and for the combined female (male) group as well, the minimum linear CM1 distance was correlated with the volume of lateral ventricles of the brain. As we can see from Table 6, the correlation between CM1 distance and volume of lateral ventricles differed by sex, being greater in men. Values of the coefficients and their significance imply a high correlation between the CM1 distance and the volume of lateral ventricles in subgroup M1 (see also Fig. 4a) as well as in M1-3 analysed as one group (see also Fig. 4b). In the female subgroup F1 we can only see a low degree of correlation between CM1 and volume of lateral ventricles (see also Fig. 4c), but this correlation was improved when F1-3 were analysed as a whole (see also Fig. 4d). When distances CM1 and CM2 were taken

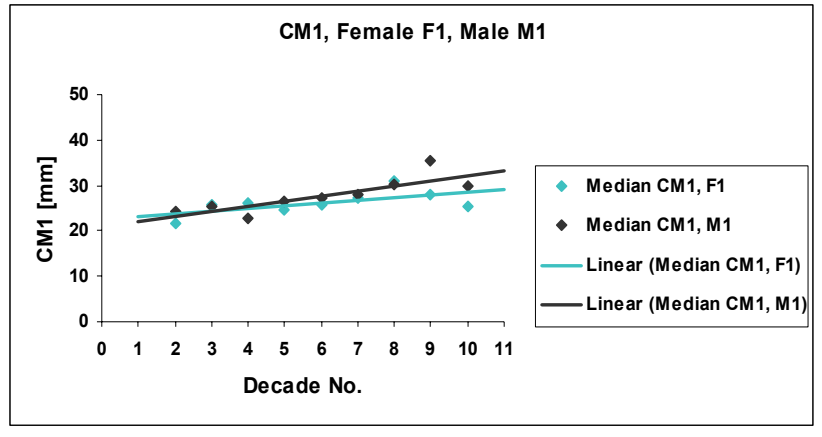

Fig. 2a. CM1 distance medians in relation to age and gender (group 1).

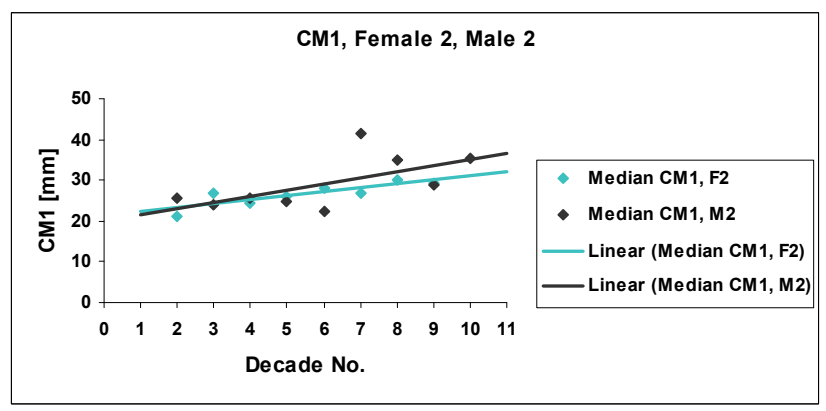

Fig. 2b. CM1 distance medians in relation to age and gender (group 2).

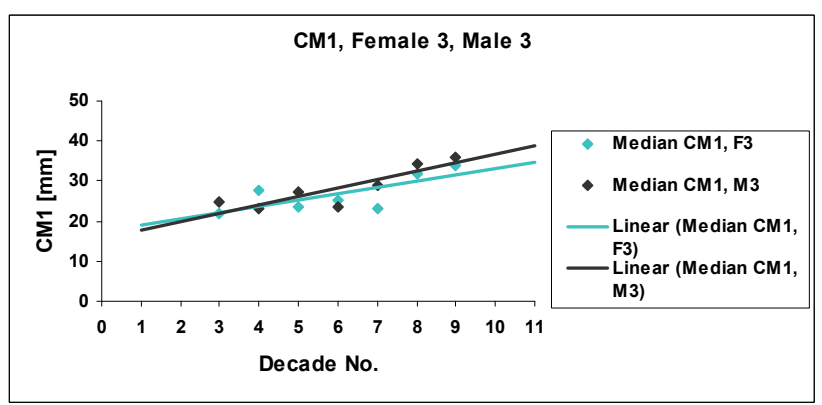

Fig. 2c. CM1 distance medians in relation to age and gender (group 3).

together and their sum was analysed for correlation with volume of brain lateral ventricles, the gender differences were noticed again. The correlation in men was found to be at a reasonable level, while that in women was found to be low (when F1-3 were analysed as a whole), in subgroup F1 alone even very low (Table 6).

\section{DISCUSSION}

Changes in brain volume have been reported to be associated with changes in size of cerebrospinal fluid spaces and, up to now, many attempts have been made to asses the morphological changes of various brain structures and compartments in humans. Autopsy studies ${ }^{11,12}$ and recently also CT and MRI studies ${ }^{13-16}$ have shown that both the brain volume and its weight decrease with advancing 
Table 1. CM1 in female subgroup 1 (F1).

\begin{tabular}{|l|c|c|r|r|r|r|r|c|r|c|}
\hline \multicolumn{7}{|c|}{ Category labels and numerical summaries for CM1 [mm] in subgroup F1 } \\
\hline Decade of age & All & 2 & \multicolumn{1}{c|}{3} & \multicolumn{1}{c|}{4} & \multicolumn{1}{c|}{5} & \multicolumn{1}{c|}{6} & \multicolumn{1}{c|}{7} & \multicolumn{1}{c|}{8} & \multicolumn{1}{c|}{9} & 10 \\
\hline Number (n) & 133 & 2 & 25 & 12 & 15 & 29 & 21 & 15 & 12 & 2 \\
\hline Mean & & 21.5 & 26.388 & 26.617 & 25.027 & 25.831 & 25.324 & 30.513 & 28.742 & 25.2 \\
\hline St Dev & & 1.131 & 3.634 & 6.276 & 3.019 & 4.586 & 4.433 & 4.719 & 3.551 & 1.414 \\
\hline Skew & & & 0.479 & 0.032 & 0.471 & 1.226 & -0.400 & -0.230 & -0.077 & \\
\hline Min & & 20.7 & 19.7 & 16.2 & 20.7 & 18.4 & 14.8 & 21.5 & 21.5 & 24.2 \\
\hline Q1 & & 20.7 & 24.2 & 21.6 & 23 & 23 & 22.6 & 28.7 & 27 & 24.2 \\
\hline Median & & 21.5 & 25.7 & 26.2 & 24.6 & 25.7 & 27.3 & 30.9 & 28 & 25.2 \\
\hline Q3 & & 22.3 & 28.3 & 32.15 & 26.6 & 27.6 & 27.6 & 32.5 & 30.75 & 26.2 \\
\hline Max & & 22.3 & 34.6 & 35.8 & 31.6 & 38.8 & 33.6 & 39.4 & 35.3 & 26.2 \\
\hline
\end{tabular}

Table 2. CM1 in male subgroup 1 (M1).

\begin{tabular}{|l|c|c|c|c|c|c|c|c|c|c|}
\hline \multicolumn{7}{|c|}{ Category labels and numerical summaries for CM1 [mm] in subgroup M1 } \\
\hline Decade of age & All & 2 & 3 & 4 & 5 & 6 & 7 & 8 & 9 & 10 \\
\hline Number (n) & 90 & 1 & 12 & 12 & 18 & 19 & 11 & 9 & 7 & 1 \\
\hline Mean & & 0 & 25.192 & 24.258 & 26.339 & 27.7 & 29.609 & 29.922 & 34.129 & 0 \\
\hline St Dev & & & 4.487 & 4.820 & 5.341 & 4.241 & 4.968 & 3.810 & 6.779 & \\
\hline Skew & & & -0.472 & 0.277 & 0.156 & 0.527 & -0.232 & -0.085 & -1.009 & \\
\hline Min & & 24.3 & 16 & 16.3 & 16.6 & 21.1 & 19.6 & 23.4 & 21.1 & 29.7 \\
\hline Q1 & & & 22.6 & 21.75 & 23.3 & 24.4 & 26.8 & 28.1 & 29.9 & \\
\hline Median & & & 25.4 & 22.85 & 26.6 & 27.2 & 28.1 & 30.2 & 35.3 & \\
\hline Q3 & & & 28.3 & 27.95 & 29.6 & 30 & 32.4 & 32.4 & 38.7 & \\
\hline Max & & 24.3 & 32.4 & 32.8 & 38.2 & 36.7 & 37.8 & 36.3 & 41.6 & 29.7 \\
\hline
\end{tabular}

Table 3. Association between CM1 distance and the decade of age.

\begin{tabular}{|c|r|c|c|c|c|}
\hline Subgroup & \multicolumn{1}{|c|}{$\mathrm{n}$} & $\begin{array}{c}\text { P of regression } \\
\text { model }\end{array}$ & Kendall's $\tau_{\mathrm{b}}$ & $95 \%$ CI for $\tau_{\mathrm{b}}$ & P for $\tau_{\mathrm{b}}$ \\
\hline F1 & 133 & 0.0202 & 0.157 & $0.044-0.271$ & 0.012 \\
\hline F2 & 33 & 0.0178 & 0.348 & $0.116-0.580$ & 0.007 \\
\hline F3 & 20 & 0.0435 & 0.243 & $(-0.111)-0.596$ & 0.165 \\
\hline M1 & 90 & $<0.0001$ & 0.328 & $0.202-0.454$ & $<0.0001$ \\
\hline M2 & 33 & 0.013 & 0.213 & $(-0.026)-0.452$ & 0.108 \\
\hline M3 & 24 & 0.0008 & 0.419 & $0.203-0.636$ & 0.008 \\
\hline
\end{tabular}


Table 4. Differences in CM1 distance between men and women.

\begin{tabular}{|c|c|}
\hline subgroups compared & $\mathrm{P}$ \\
\hline F1 and M1 & 0.156 \\
\hline F2 and M2 & 0.461 \\
\hline F3 and M3 & 0.320 \\
\hline
\end{tabular}

age. Results obtained from autopsy studies have been reported to be subject to post mortem or fixation changes of biological material. The association between cerebral atrophy and increasing age has been documented in papers published e.g. by Matsumae et al. ${ }^{17}$ and Resnick et al. ${ }^{18}$ Studies based on CT and MRI examination also used ratios calculated from linear dimensions ${ }^{19,20}$. However, the use of ratios in research based on imaging techniques may represent a serious problem resulting from the fact that the size of both structures is subject to error. This causes that final result has two sources of error ${ }^{21}$. That is why we focus on direct linear (or volumetric) parameters first of all. Within the scope of our research intention our aim was to identify pairs of parameters with the tightest correlation between the volume of lateral ventricles and brain or intracranial linear parameters, because volumetric measurements are time consuming to be used in daily practice.

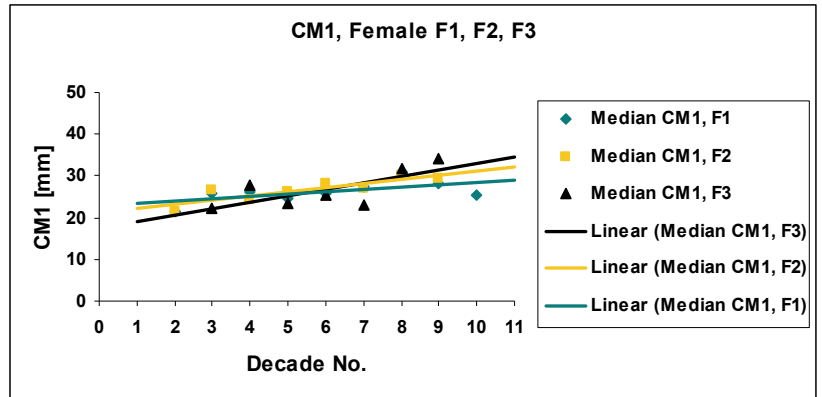

Fig. 3a. CM1 distance medians in relation to age. Three subgroups of women - F1, F2, F3.

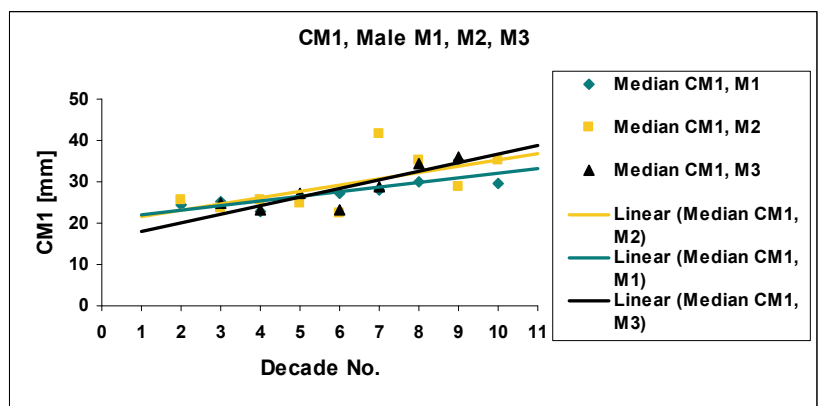

Fig. 3b. CM1 distance medians in relation to age. Three subgroups of men - M1, M2, M3.

Table 5. Pair-wise comparisons across predefined subgroups of women and men (Tukey method).

\begin{tabular}{|c|c|c|c|}
\hline \multicolumn{2}{|c|}{ Women $(\mathrm{n}=186)$} & \multicolumn{2}{c|}{ Men $(\mathrm{n}=147)$} \\
\hline compared subgroups & $\mathrm{P}$ & compared subgroups & $\mathrm{P}$ \\
\hline F1 and F3 & 0.705 & M1 and M3 & 0.554 \\
\hline F2 and F3 & 0.857 & M2 and M3 & 0.999 \\
\hline F1 and F2 & 0.960 & M1 and M2 & 0.531 \\
\hline
\end{tabular}

Table 6. Correlation between parameters in the left column and the respective volume of brain lateral ventricles.

\begin{tabular}{|l|c|c|c|}
\hline Parameters (subgroups) & $\mathrm{r}$ & $95 \% \mathrm{CI}$ for $\mathrm{r}$ & P for $\mathrm{r}$ \\
\hline CM1 (F1) & $\mathbf{0 . 3 5 7}$ & $0.198-0.497$ & $<0.0001$ \\
\hline CM1 (F1-3) & $\mathbf{0 . 4 6 5}$ & $0.344-0.571$ & $<0.0001$ \\
\hline CM1 (M1) & 0.659 & $0.523-0.762$ & $<0.0001$ \\
\hline CM1 (M1-3) & 0.675 & $0.576-0.754$ & $<0.0001$ \\
\hline CM1+CM2 (F1) & $\mathbf{0 . 1 1 2}$ & $(-0.059)-0.277$ & 0.1975 \\
\hline CM1+CM2 (F1-3) & $\mathbf{0 . 2 1 2}$ & $0.071-0.346$ & 0.0035 \\
\hline CM1+CM2 (M1) & 0.434 & $0.250-0.588$ & $<0.0001$ \\
\hline CM1+CM2 (M1-3) & 0.461 & $0.323-0.579$ & $<0.0001$ \\
\hline
\end{tabular}




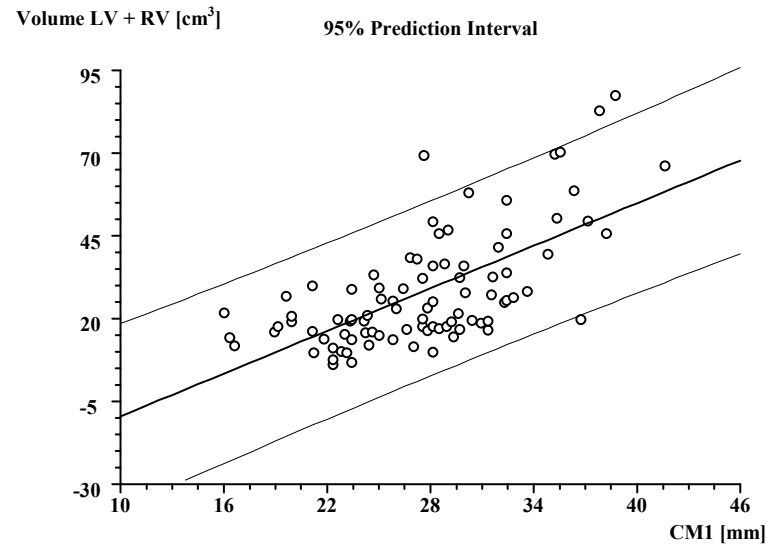

Fig. 4a. The CM1 distance correlated with volume of lateral ventricles, subgroup M1.

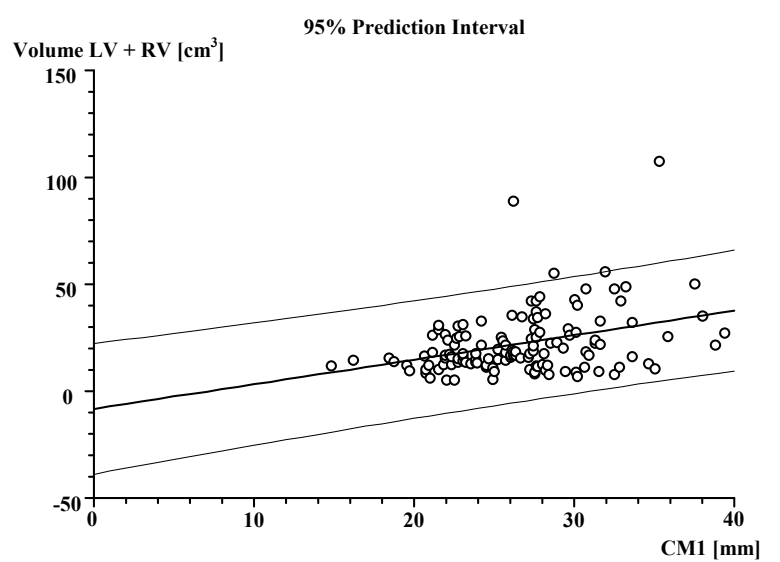

Fig. 4c. The CM1 distance correlated with volume of lateral ventricles, subgroup F1.

This study gives a detailed analysis of an association between the cella media distance (CM1) derived from CT imaging for a series of three on-diagnoses based groups of right-handed individuals between ages 18-96 years and the following parameters: decade of age, gender and volume of lateral ventricles. The cella media distance was found to increase significantly with age in all the studied subgroups (F1, F2, F3, M1, M2, and M3). As it could be seen from the graphs, a visual comparison of the increase in cella media distance between women and men within the individual groups 1, 2 and 3, the "male" lines have higher slopes than the "female" ones. The difference in CM1 distance between the gender subgroups was not significant in any of the three groups. However, the age-associated increase in the CM1 distance appears to be more pronounced in the male than in the female subjects. This finding should be kept in mind when comparing associations between brain volume, single brain compartments volumes or other brain linear measures and decades of age. For example Condon et al. ${ }^{22}$ found a significant decrease in the normalized brain volume between the ages of 20 and 60 years in males by $1.6 \%$ per decade while in females of the same age the decrease was less $(0.5 \%)$ and was not confirmed as statistically significant.

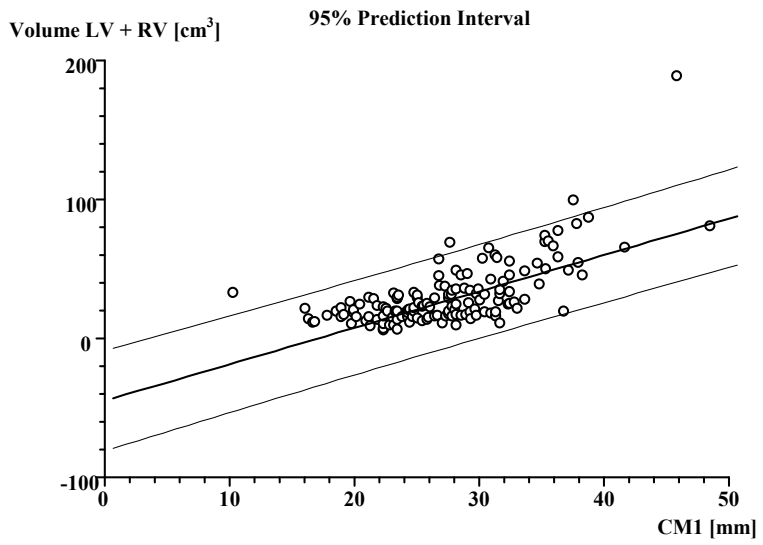

Fig. 4b. The CM1 distance correlated with volume of lateral ventricles, subgroups M1-3.

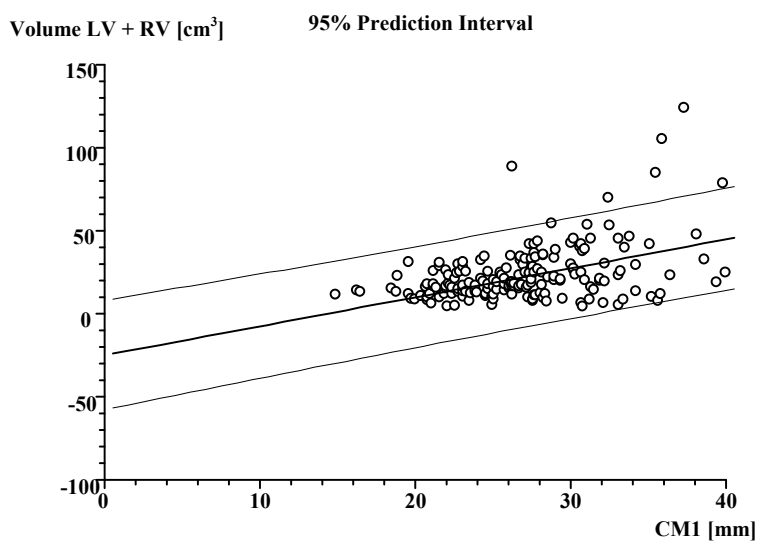

Fig. 4d. The CM1 distance correlated with volume of lateral ventricles, subgroups F1-3.

In addition, one more difference between the analysed gender groups ( $\mathrm{F}$ and $\mathrm{M}$ ) was found in our study: the correlation between cella media distance (CM1) and volume of lateral ventricles was found to be greater in men (M) in both the subgroup M1 $(r=0.659, \mathrm{P}<0.0001)$ and in the mixed group where all the three male subgroups differing in diagnoses (M1, M2 and M3) were combined and analysed as one group M1-3 ( $\mathrm{r}=0.675, \mathrm{P}<0.0001)$. On the other hand, in the female group (F), the correlation was lower, however, still significant (with $r=0.357$, $\mathrm{P}<0.0001$ for $\mathrm{F} 1$, and $\mathrm{r}=0.465, \mathrm{P}<0.0001$ for $\mathrm{F} 1-3)$. The last finding was that the correlation between CM1 and volume of lateral ventricles seems to be independent of the three predefined groups assigned in this study. Nevertheless, we should keep in mind that the subgroups M1 and F1 were more numerous than subgroups F2, F3, M2 and M3 - thus, the correlation coefficients might not be confirmed as significant in F3 and M2 due to low numbers of subjects involved. In the present study, the cella media distance was not significantly larger either in F2 (M2) or F3 (M3) than those in F1 (M1). However, the graphs indicate that the largest increase in CM1 distance could be anticipated in the subgroups F3 and M3. 


\section{CONCLUSIONS}

In our study, the cella media distance (CM1) appears to be highly correlated with the volume of cerebral lateral ventricles in the group of men and thus it might be used as a predictor of age-dependent changes in lateral ventricular volume in men. On the other hand, in the group of women the correlation between CM1 distance and the volume of lateral ventricles was found to be low (F1) or reasonable (F1-3). In comparisons made separately for female and male subgroups no statistically significant differences in cella media distance were found between the three predefined groups 1,2 and 3 .

\section{ACKNOWLEDGEMENTS}

This work was supported by VEGA, the Grant Agency of Ministry of Education of the Slovak Republic, Grants No. 1/4265/07, 1/0293/08.

\section{REFERENCES}

1. Soininen H, Puranen M, Riekkinen PJ. Computed Tomography Findings in Senile Dementia and Normal Aging. J Neurol Neurosurg \& Psychiatry 1982; 45:50-54.

2. Van Horne JD, McManus IC. Ventricular Enlargement in Schizophrenia: A Meta-Analysis of Studies of the Ventricle:Brain Ratio (VBR). British Journal of Psychiatry 1992; 160:687-697.

3. Stober T, Wussow W, Schimrigk K. Bicaudate Diameter - the most specific and simple CT parameter in the diagnosis of Huntington's disease. Neuroradiology 1984; 26:25-28.

4. Zhang Y, Londos L, Minthon C, Wattmo C, Liu H, Aspelin P, et al. Usefulness of Computed Tomography Linear Measurenent in Diagnosing Alzheimer's Disease. Acta Radiologica 2008; 49(1):91-97.

5. Kruggel F. MRI-Based Volumetry of Head Compartments: Normative Values of Healthy Adults. Neuroimage 2006; 30:1-11.

6. Blatter DD, Bigler ED, Gale SD, Johnson SC, Andersen CV, Burnett BM, et al. Quantitative Volumetric Analysis of Brain MR: Normative Database Spanning 5 Decades of Life. Am J Neuroradiol 1995; 16:241-251.

7. Courchesne E, Chisum HJ, Townsend J, Cowles A, Covington J, Egaas B, et al. Normal Brain Development and Aging: Quantitative Analysis at in Vivo MR Imaging in Healthy Volunteers. Radiology 2000; 216(3):672-682.
8. Gomori JM, Steiner I, Melamed E, Cooper G. The Assesment of Changes in Brain Volume Using Combined Linear Measurement. A CT-Scan Study. Neuroradiology 1984; 26:21-24.

9. Kiroğlu Y, Karabulut N, Oncel C, Yagci B, Sabir N, Ozdemir B. Cerebral Lateral Ventricular Asymmetry on CT: How much Asymmetry is Representing Pathology? Surg. Radiol. Anat. 2008; 30:249-255.

10. Aylward EH, Schwartz J, Machlin S, Pearlson G. Bicaudate Ratio as a Measure of a Caudate Volume on MR Images. Am J Neuroradiol 1991; 12:1217-1222.

11. Bull JDW. The Robert Wartenberg Memorial Lecture. The Volume of the Cerebral Ventricles. Neurology 1961; 11:1-10.

12. Miller AKH, Alston RL, Corsellis JAN. Variation with Age in the Volumes og Grey and White Matter in the Cerebral Hemispheres of Man: Measurements with an Image Analyser. Neuropathology and Applied Neurobiology 1980; 6:119-132.

13. Bartholomeusz HH, Courchesne E, Karns CM. Relationship Between Head Circumference and Brain Volume in Healthy Normal Toddlers, Children, and Adults. Neuropediatrics 2002; 33:239-241.

14. Cramer GD, Allen DJ, DiDio LJA. Volume Determination of the Encephalic Ventricles with CT and MRI. Surg Radiol Anat 1990; 12:59-64.

15. Cramer GD, Allen DJ, DiDio LJA, Potvin W, Brinker R. Evalustion of Encephalic Ventricular Volume from the Magnetic Resonance Imaging Scans of Thirty-Eight Human Subjects. Surg Radiol Anat 1990; 12:287-290.

16. Cramer GD, Allen DJ, DiDio LJA, Potvin W, Brinker R. Comparison of Computerized Tomography with Magnetic Resonance Imaging (MRI) in the Evaluation of Encephalic Ventricular Volume. Surg Radiol Anat 1990; 12:135-141.

17. Matsumae M, Kikinis R, Mórocz IA, Lorenzo AV, Sándor T, Albert MS, et al. Age-Related Changes in Intracranial Compartments Volumes in Normal Adults Assesed by Magnetic Resonance Imaging. J Neurosurg 1996; 84:982-991.

18. Resnick SM, Pham DL, Kraut MA, Zonderman AB, Davatzikos C. Longitudinal Magnetic Resonance Imaging Studies of Older Adults: A Schrinking Brain. The Journal of Neuroscience 2003; 23(8):3295-3301.

19. Barron SA, Jacobs L, Kinkel WR. Changes in Size of Normal Lateral Ventricles During Aging Determined by Computerized Tomography. Neurology 1976; 25:1011-1013.

20. Brinkman SD, Sarwar M, Levin HS, Morris HH. Quantitative Indexes of Computed Tomography in Dementia and Normal Aging. Radiology 1981; 138:89-92.

21. Arndt S, Cohen G, Alliger RJ, Swayze VW, Andreasen NC. Problems with Ratio and Proportions Measures of Imaged Cerebral structures. Psychiatry Research: Neuroimaging 1991; 40:79-89.

22. Condon B, Grant R, Hadley D, Lawrence A. Brain and Intracranial Cavity Volumes: in vivo determination by MRI. Acta Neurol Scand 1988; 78:387-393. 
